\title{
Cardiac Device Oversensing without Shock or Anti-Tachycardia Pacing
}

National Cancer Institute

\section{Source}

National Cancer Institute. Cardiac Device Oversensing without Shock or Anti-Tachycardia

Pacing. NCl Thesaurus. Code C99928.

This manifests as sensing of non-cardiac depolarization signals that did not meet

arrhythmia detection criteria and do not elicit programmed tachyarrhythmia therapy.

(ACC) 